\title{
Acne Scarring: Why We Should Act Sooner Rather Than Later
}

Brigitte Dréno · Linda Stein Gold

Received: April 27, 2021 / Published online: June 11, 2021

(C) The Author(s) 2021

Keywords: Acne vulgaris; Inflammation; Acne scarring

\section{Key Summary Points}

Acne scarring can affect any patient with acne, regardless of severity, and place a considerable psychological and psychosocial burden on their lives.

Recent studies have shed new light on the pathophysiology of acne and acne scarring, confirming the role of family history and the importance of early and sustained treatment of inflammation for acne scar prevention in all patients.

\section{B. Dréno}

Department of Dermatology, CHU Nantes, CIC

1413, CRCINA, University of Nantes, Nantes, France

L. Stein Gold

Clinical Research Division, Head of Dermatology,

Henry Ford Hospital, Detroit, MI, USA

\section{Stein Gold $(\bowtie)$}

Henry Ford Health System, 6530 Farmington Rd, West Bloomfield Township, Detroit, MI 48322, USA e-mail: LSTEIN1@hfhs.org
For many years we have relied on invasive, physical treatments for acne scars but we now have topical anti-inflammatory treatments that can reduce the risk of acne scarring, including 0.3\% adapalene $/ 2.5 \%$ benzoyl peroxide.

Current challenges in acne scar prevention include a lack of open communication between patients and physicians and poor treatment adherence.

Ultimately, we must consider and inform our patients of their risk of acne scarring in our initial treatment plans and treat acne inflammation early and effectively to achieve the best outcomes.

\section{DIGITAL FEATURES}

This article is published with digital features, including a summary slide, to facilitate understanding of the article. To view digital features for this article go to https://doi.org/10.6084/ m9.figshare.14679816.

\section{COMMENTARY}

Acne of any severity can lead to acne scarring, yet historically acne scars have only been 
considered as a consequence of severe or very severe acne [1]. Indeed, among people with acne scars, nearly a third have almost clear/mild acne and $40 \%$ have moderate acne $[1,2]$. Acne scarring can reduce quality of life, and places a significant psychological and psychosocial burden on patients, including a lack of self-confidence and concerns over body image [1, 2]. Thus, we must consider all patients, even those with mild acne, for their risk of acne scarring and provide early and sustained treatment to prevent the long-term consequences [1]. Here, we discuss acne scar risk assessment approaches, our new understanding of the pathophysiology of acne scarring, and the importance of open communication and treatment adherence in prevention strategies.

Currently, the risk of acne scarring is not routinely assessed in all patients with acne during consultations. Although the prevalence of acne scarring is higher in those with severe acne, physicians must thoroughly evaluate all patients for scarring, as even those with superficial lesions may be at risk [1]. The most important risk factors are a family history of acne scarring and delayed treatment of acne lesions. Practical risk assessment tools such as Tan et al.'s four-item, self-administered patient questionnaire may help identify those at risk of atrophic acne scars and in need of rapid and effective treatment. This evidence-based tool considers four risk factors: worst ever severity of acne, duration of acne, family history of atrophic acne scars, and lesion manipulation behaviors [2]. Moreover, the facial acne scar evaluation tool (FASET) is a simple and reproducible method to assess existing scars, incorporating three domains: scar counts (2-4 mm, > $4 \mathrm{~mm})$, overall global severity rating, and the concept of area of involvement (scar dispersion) [3].

In our experience, the best approach for risk assessment is to holistically evaluate patients in person, where possible, considering all acne lesions on a clean, makeup-free face. Recently, teledermatology has gained traction; however, it may be difficult to conduct an accurate assessment of the skin, including the inflammation profile, because of poor lighting and video quality in teleconsultations. Thus, telexpertise based on secure emails with patient photographs can be useful for evaluating patients over time, especially in the absence of face-to-face consultations during the COVID-19 pandemic. However, as photographs cannot depict the three-dimensional nature of some acne lesions and scars, they should not replace face-to-face consultations where possible.

Recent findings have shed new light on the pathophysiology of acne and acne scarring. Atrophic acne scars form in a continuous process, primarily evolving from inflammatory (papules) and post-inflammatory lesions [4]. It is worth noting that in our experience, there are two schools of thought regarding macular erythema: some dermatologists believe it is a transient inflammatory state associated with the healing process, whereas others consider it a precursor to acne scarring. The duration of papules is a key driver of scar risk and long-lived papules are characterized by a $\mathrm{B}$ cell $\left(\mathrm{CD} 20^{+}\right)$ infiltrate [5]. There is an important link between the duration and severity of inflammation, loss of lipid-associated gene expression, and altered sebaceous gland structure (via apoptosis and necrosis), which leads to atrophic scars $[4,5]$.

Recently, Josse et al. showed bacterial colonization in microcomedones, confirming the role of bacteria early in the development of acne lesions [6]. During lesion development, the proinflammatory profile of Cutibacterium acnes in normal skin changes as a result of the activation of virulence genes [7]. C. acnes modifies the sebum via bacterial metabolism, secreting enzymes that produce pro-inflammatory freefatty acids, which kick-start the inflammatory process [6]. There is also a loss of balance between the different $C$. acnes phylotypes, and a dysbiosis of the skin microbiome (involving Staphylococcus epidermidis and C.acnes) in the superficial dermis, which contribute to inflammation. Evidence suggests that restoring microbial diversity may prevent the abnormal healing of acne lesions, highlighting the need for anti-inflammatory treatments that modify the skin microbiome [8].

It is important to maintain a balance between matrix metalloproteinases and their inhibitors as well as pro-inflammatory and antiinflammatory cytokines to avoid acne scarring. 
Currently, the best way to achieve this aim is via early, effective, and sustained treatment of acne lesions [4]. We typically aim to achieve greater than $50 \%$ clearance with initial treatment, followed by topical agents as maintenance therapy to avoid relapse. Notably, the risk of acne scarring is not limited to adolescence, as acne (and thus acne scarring) can persist or recur in adulthood. For example, face-mask-induced acne ("maskne") is becoming prevalent among adults during the COVID-19 pandemic. This emphasizes the importance of encouraging maintenance therapy, particularly as patients may find it difficult to adhere to their treatment for more than 6 months.

Until now, we have typically relied on invasive, physical treatments for acne scarring. For the first time, we have evidence for a new, preventative approach with a topical agent, $0.3 \%$ adapalene/2.5\% benzoyl peroxide (A/BPO), which demonstrated a reduction in atrophic scars over 6 months in a randomized, vehiclecontrolled, split-face, clinical study [9]. Both $0.1 \% \mathrm{~A} / \mathrm{BPO}$ and $0.3 \% \mathrm{~A} / \mathrm{BPO}$ are important non-invasive and effective acne treatments, which can be used in patients with any acne severity $[9,10]$. In line with current guidelines, we generally consider topical retinoids or fixed combinations for the treatment of mild to moderate acne, and a combination regimen of topical treatments with antibiotics or isotretinoin for more severe acne or nodular lesions, respectively. However, these preventative strategies do not apply to excoriated lesions; the healing process for mechanical manipulation is distinct and so we must continue to deter patients from excoriation from a young age.

Key challenges in the prevention of acne scarring include limited time during consultations, lack of open physician-patient communication, and poor treatment adherence. Patients with acne scars experience a psychological burden, and are typically discouraged by the fact that their existing scars cannot be treated. It is important to create a comfortable environment for patients to discuss their acne scars and provide hope for prevention of future scarring. Some patients are deterred by the need for long-term treatment and thus do not initiate the conversation during consultations. Educating patients on the basic pathophysiology of acne scarring may motivate them to act and prevent further scars appearing in the future. For example, using a simple visual tool or diagram illustrating the role of inflammation and bacteria in the development of acne scarring may help patients understand the need for early and sustained treatment. We appreciate that the knowledge transfer from physician to patient can be difficult; nurses can play an important role in patient education, particularly when there is limited time during consultations, and influencers on social media platforms can be a useful reference/role model, particularly for younger patients.

Ultimately, there has been significant progress in the field in recent years leading to an improved understanding of the underlying pathophysiology of acne scarring, including the implication of bacterial colonization of microcomedones [4-8]. These findings confirm the importance of thorough evaluation and early and sustained treatment of inflammation for acne scar prevention in all patients regardless of their acne severity. Thus, we must collectively consider the risk of acne scarring in our initial treatment plans and encourage treatment adherence to achieve the best outcomes for our patients.

\section{ACKNOWLEDGEMENTS}

Funding. Galderma funded the Rapid Service Fee and medical writing support.

Medical Writing and/or Editorial Assistance. Medical writing support was provided by Dr Arti Sikka (Publicis Langland).

Authorship. All named authors meet the International Committee of Medical Journal Editors (ICMJE) criteria for authorship for this article, take responsibility for the integrity of the work as a whole, and have given their approval for this version to be published. 
Authorship Contributions. Prof. Brigitte Dréno and Dr Linda Stein Gold drafted the manuscript.

Disclosures. Prof. Brigitte Dréno is an advisory board member for Galderma, Novartis, and Leo Pharma. Dr Stein Gold is an investigator and/or consultant and/or speaker for Galderma, Ortho Dermatologics, Sun, Vyne, Sol Gel, Almirall, and Novartis.

Compliance with Ethics Guidelines. This article is based on previously conducted studies and does not contain any new studies with human participants or animals performed by any of the authors.

Open Access. This article is licensed under a Creative Commons Attribution-NonCommercial 4.0 International License, which permits any non-commercial use, sharing, adaptation, distribution and reproduction in any medium or format, as long as you give appropriate credit to the original author(s) and the source, provide a link to the Creative Commons licence, and indicate if changes were made. The images or other third party material in this article are included in the article's Creative Commons licence, unless indicated otherwise in a credit line to the material. If material is not included in the article's Creative Commons licence and your intended use is not permitted by statutory regulation or exceeds the permitted use, you will need to obtain permission directly from the copyright holder. To view a copy of this licence, visit http://creativecommons.org/licenses/bync/4.0/.

\section{REFERENCES}

1. Dréno B, Layton A, Bettoli V, Lozada VT, Kang S. Evaluation of the prevalence, risk factors, clinical characteristics, and burden of acne scars among active acne patients in Brazil, France, and the USA. J Am Acad Dermatol. 2017;76:AB132.

2. Tan J, Thiboutot D, Gollnick H, et al. Development of an atrophic acne scar risk assessment tool. J Eur Acad Dermatol Venereol. 2017;31:1547-54.

3. Dréno B, Tan J, Layton A, et al. New evidence-based facial acne scar evaluation tool (FASET) to assess atrophic scars. J Am Acad Dermatol. 2015;72: AB435.

4. Tan J, Bourdés V, Bissonnette R, et al. Prospective study of pathogenesis of atrophic acne scars and role of macular erythema. J Drugs Dermatol. 2017;16:566-72.

5. Carlavan I, Bertino B, Rivier M, et al. Atrophic scar formation in patients with acne involves long-acting immune responses with plasma cells and alteration of sebaceous glands. Br J Dermatol. 2018;179: 906-17.

6. Josse G, Mias C, Le Digabel J, et al. High bacterial colonization and lipase activity in microcomedones. Exp Dermatol. 2020;29:168-76.

7. Dréno B, Pécastaings S, Corvec S, et al. Cutibacterium acnes (Propionibacterium acnes) and acne vulgaris: a brief look at the latest updates. J Eur Acad Dermatol Venereol. 2018;32(Suppl 2):5-14.

8. Dréno B, Dagnelie MA, Khammari A, Corvec S. The skin microbiome: a new actor in inflammatory acne. Am J Clin Dermatol. 2020;21:18-24.

9. Dréno B, Bissonnette R, Gagné-Henley A, et al. Prevention and reduction of atrophic acne scars with adapelene $0.3 \%$ /benzoyl peroxide $2.5 \%$ gel in subjects with moderate or severe facial acne: results of a 6-month randomized, vehicle-controlled trial using intra-individual comparison. Am J Clin Dermatol. 2018;19:275-86.

10. Dréno B, Tan J, Rivier M, Martel P, Bissonnette R. Adapalene $0.1 \%$ /benzoyl peroxide $2.5 \%$ gel reduces the risk of atrophic scar formation in moderate inflammatory acne: a split-face randomized controlled trial. J Eur Acad Dermatol Venereol. 2017;31:737-42. 